\title{
D-instantons in real time dynamics
}

\author{
Si-wen $\mathrm{Li}^{1, * *}$ and Shu $\mathrm{Lin}^{2, \dagger}$ \\ ${ }^{1}$ Department of Physics, Center for Field Theory and Particle Physics, \\ Fudan University, Shanghai 200433, China \\ ${ }^{2}$ School of Physics and Astronomy, Sun Yat-Sen University, Zhuhai 519082, China
}

(Received 15 April 2018; published 11 September 2018)

\begin{abstract}
An instanton is known to exist in Euclidean spacetime only. Their role in real time dynamics is usually understood as a tunneling effect by Wick rotation. We illustrate other effects of instanton in holography by investigating five-dimensional effective gravity theory of the black D3-brane-D-instanton system. The supergravity description of the D3-brane-D-instanton system is dual to the super-Yang-Mills theory with topological excitations of the vacuum. We obtain Euclidean correlators in the presence of instantons by analyzing the fluctuations of the bulk fields in the five-dimensional effective theory. Furthermore, analytic continuation of Euclidean correlators leads to retarded correlators, which characterize real time dynamics. We find interestingly real time fluctuations of topological charge can destroy instantons and the lifetime of instanton is set by temperature. This implies instanton contribution to "real time dynamics" is suppressed at high temperature, which is analogous to classic field theory results that the instanton contribution to "thermodynamics" is suppressed at high temperature.
\end{abstract}

DOI: 10.1103/PhysRevD.98.066002

\section{INTRODUCTION}

Instantons in quantum chromodynamics (QCD) are known as topologically nontrivial excitations of the vacuum. They are known to contribution to the thermodynamics of QCD and are closely related to chiral symmetry breaking. We refer the readers to $[1,2]$ for comprehensive reviews. Instantons are also known to consist of constituents called BPS (Bogomol'nyi-Prasad-Sommerfield) monopoles or dyons. There have been continuous efforts in linking confinement and chiral symmetry breaking with instanton constituents [3-11]. While extensive literature focuses on the role of instantons in thermodynamics and phase transition, their role in real time dynamics has not received enough attention. The goal of this work is to fill the gap. Similar to instantons in quantum mechanics, instantons in QCD are generally viewed as tunneling processes. A typical question one may ask is how does the presence of an instanton affects certain correlation functions, e.g., a retarded correlator $G^{R}(\omega)$, which is a genuine real time quantity. It is known that the retarded correlator is related to the Euclidean counterpart $G^{E}\left(\omega_{E}\right)$ by

\footnotetext{
siwenli@fudan.edu.cn

linshu8@mail.sysu.edu.cn
}

Published by the American Physical Society under the terms of the Creative Commons Attribution 4.0 International license. Further distribution of this work must maintain attribution to the author(s) and the published article's title, journal citation, and DOI. Funded by SCOAP ${ }^{3}$.

$$
G^{E}\left(\omega_{E}\right)=-G^{R}(\omega),
$$

with $\omega=i \omega_{E}=2 i \pi T n$. Note that the equality holds only when $\omega_{E}$ takes value on discrete Matsubara frequencies. In order to obtain the retarded correlator with an arbitrary real $\omega$, analytic continuation is needed. However, it is not always well defined. For example, in lattice gauge theory, $G^{E}$ and $G^{R}$ are related through spectral function. Going from $G^{E}$ to $G^{R}$ involves an ambiguity.

Holography provides us with a possibility to study the effect of instantons in real time dynamics. Instantons in holographic models have been constructed as a D-instanton background [12-14]. The effects of instantons on chiral symmetry breaking and heavy quark potential have been discussed holographically in [15-17]. The thermodynamics of anisotropic instanton distribution has been studied in $[18,19]$. While these are essentially related to Euclidean quantities, holography also allows for the easy access of real time quantities. In holography, we may also work with purely imaginary $\omega_{E}$, which can be viewed as a natural analytic continuation of (1.1). Such an approach allows for straightforward extraction of a retarded correlation function from (1.1). In this paper, we illustrate the effect of instantons on retarded correlators of a topological charge at finite temperature, which are measures of its fluctuations. Since instantons themselves carry a topological charge, we find, interestingly, the fluctuations can destroy instantons in real time. The lifetime of an instanton is found to be set by the temperature. Simply speaking, the contribution of an instanton to "real time dynamics" is 
suppressed at high temperature. This is in line with the classic field theory results: the contribution of an instanton to "thermodynamics" is suppressed at high temperature [20,21].

The paper is organized as follows: in Sec. II, we review the D3-brane-D-instanton background at finite temperature. In Sec. III, we obtain the five-dimensional (5D) effective action and study 5D fluctuations in the D-instanton background. We proceed to calculate two point correlators of stress tensor components, the gluon condensate, and topological charge in Sec. IV. Finally, we discuss the effect of instantons in real time dynamics and conclude in Sec. V.

\section{REVIEW OF D3-BRANE-D-INSTANTON SYSTEM}

In this section, let us briefly review the background of the black D3-branes with D-instantons and its dual field theory. The original example for D3-branes with D-instantons was proposed in [12], which is a deformed D3-brane solution of type IIB supergravity by a nontrivial scalar field. In order to preserve $1 / 2$ of supersymmetry, a Ramond-Ramond (RR) scalar charge is switched on and balanced by the dilaton charge in this system. The resulting solution represents a marginal "bound state" of D3-branes with smeared D-instantons, i.e., D(-1)-branes. In this paper, we focus on a finite-temperature extension of the $\mathrm{D} 3-\mathrm{D}(-1)$ background in Euclidean signature [14]. In this background there is a RR four-form $C_{4}$ and zero-form $\mathcal{C}$ field which couples to $\mathrm{D} 3$ and D(-1)-branes respectively. In the Einstein frame, the 10dimensional (10D) supergravity action is given as [22,23]

$$
\begin{aligned}
S_{10 \mathrm{D}}= & \frac{1}{2 \kappa_{10}^{2}} \int d^{10} x \sqrt{g} \\
& \times\left[\mathcal{R}_{10}-\frac{1}{2}(\partial \Phi)^{2}+\frac{1}{2} e^{2 \Phi}(\partial \chi)^{2}-\frac{1}{2}\left|F_{5}\right|^{2}\right],
\end{aligned}
$$

where $\Phi$ is the dilaton filed, and $F_{5}=d C_{4}$ is the field strength of $C_{4}$. The original RR zero-form $\mathcal{C}$ is defined as $\chi=i \mathcal{C}$ where $\chi$ is usually named as an axion. By setting $\chi=-e^{-\Phi}+\chi_{0}$ where $\chi_{0}$ is a constant, the dilaton term cancels the axion term in (2.1) so that the dynamics in the action involves the metric and the RR four-form $C_{4}$ only. The solution in Euclidean signature reads [14,23]

$$
\begin{aligned}
d s_{10}^{2} & =\frac{r^{2}}{R^{2}}\left[f(r) d \tau^{2}+\delta_{i j} d x^{i} d x^{j}\right]+\frac{1}{f(r)} \frac{R^{2}}{r^{2}} d r^{2}+R^{2} d \Omega_{5}^{2}, \\
f(r) & =1-\frac{r_{T}^{4}}{r^{4}}, \quad \chi=-e^{-\Phi}+\chi_{0}, \\
e^{\Phi} & =1+\frac{q}{r_{T}^{4}} \log \left[\frac{1}{f(r)}\right], \quad F_{5}=\sqrt{-2 \Lambda}\left(\epsilon_{5}+\star \epsilon_{5}\right) .
\end{aligned}
$$

Here $R^{4}=4 \pi g_{s} N_{c} l_{s}^{4},\left|F_{5}\right|^{2}=\frac{1}{5 !} F_{M N K P Q} F^{M N K P Q}, \tau$ is the Euclidean time defined as $\tau=i t$ and $\epsilon_{5}$ is the five-form volume element and “ $\star$ " represents the Hodge dual. The constant $q$ denotes the number density of $\mathrm{D}(-1)$-branes, i.e., D-instantons. While the dilaton becomes divergent at the black hole horizon, it does not give any effects about the thermodynamics since the bulk observables should be computed in the Einstein frame where the onshell action is the same as the case of the purely black D3-brane.

According to the AdS/CFT dictionary, the above supergravity solution holographically describes the Euclidean $\mathcal{N}=4$ super-Yang-Mills theory with $S U(N)$ gauge symmetry in a certain nonvacuum state. Hence, the constant $q$ represents the vacuum expectation value (VEV) in Euclidean spacetime:

$$
\begin{aligned}
& O_{E}=\left\langle\operatorname{Tr} \mathcal{F}^{2}\right\rangle, \quad \tilde{O}_{E}=\langle\operatorname{Tr} \mathcal{F} \wedge \mathcal{F}\rangle, \\
& O_{E}=-\tilde{O}_{E} \propto q \neq 0 .
\end{aligned}
$$

Here we define gluon condensate $O_{E}$ and topological charge density $\tilde{O}_{E}$ in terms of the Euclidean gauge field strength $\mathcal{F}$. We use subscript $E$ to indicate that the Euclidean signature is to be used. Note that the sign corresponds to that of an anti-instanton. Below we will loosely refer to this as an instanton, which does not affect the conclusion of this paper.

The temperature of the dual field theory is given by $T=\frac{r_{T}}{\pi R^{2}}$. The nontrivial profiles of $\Phi$ and $\chi$ correspond to an effective $\theta$ term. To see this, we consider the action of probed D3-branes with smeared D(-1)-branes,

$S_{D 3}=S_{D B I}+\mu_{3} \int C_{4}+\frac{i}{2} \mu_{3}\left(2 \pi \alpha^{\prime}\right)^{2} \int \chi \mathcal{F} \wedge \mathcal{F}+\cdots$,

where $\mu_{3}=\frac{1}{(2 \pi)^{3} l_{s}^{4}}$ and $\alpha^{\prime}=l_{s}^{2}$. The super-Yang-Mills action in the dual theory comes from the leading order expansion of the Dirac-Born-Infield (DBI) action in (2.4). Since $\chi$ only depends on $r$ according to (2.2), the last term in (2.4) give rises to a $\theta$ term

$$
\begin{aligned}
\chi(r) & \sim \theta(r), \\
\int_{\mathbb{R}^{4}} \chi \mathcal{F} \wedge \mathcal{F} & =\chi \int_{\mathbb{R}^{4}} \mathcal{F} \wedge \mathcal{F} \sim \theta \int_{\mathbb{R}^{4}} \mathcal{F} \wedge \mathcal{F} .
\end{aligned}
$$

This allows us to dial instanton density $q$ as an independent parameter.

\section{THE FIVE-DIMENSIONAL EFFECTIVE THEORY}

\section{A. Dimensional reduction}

In this section we are going to obtain a $5 \mathrm{D}$ effective gravity theory of the D3-D(-1) system and explore the 5D fluctuations of the bulk fields. Let us start with the 10D 
equations of motions for the bulk fields, which can be derived by varying the action $(2.1){ }^{1}$

$$
\begin{aligned}
& \mathcal{R}_{M N}=\frac{1}{2} \partial_{M} \Phi \partial_{N} \Phi-\frac{1}{2} e^{2 \Phi} \partial_{M} \chi \partial_{N} \chi+\frac{1}{6} F_{M K P Q L} F_{N}^{K P Q L}, \\
& \nabla^{2} \Phi=-e^{2 \Phi}(\partial \chi)^{2}, \quad g^{M N} \nabla_{M}\left(e^{2 \Phi} \partial_{N} \chi\right)=0
\end{aligned}
$$

where the indices $M, N, P, Q, L$ runs from 0 to 9 . Inserting the solution (2.2) into (3.1), we assume all the functions only depend on the 5D coordinates $x^{a}=\left\{x^{\mu}, r\right\}$ where $\mu=0,1,2,3$. Hence, (3.1) can be rewritten as

$$
\begin{aligned}
\mathcal{R}_{a b}^{(5 \mathrm{D})} & =\Lambda g_{a b}+\frac{1}{2} \partial_{a} \Phi \partial_{b} \Phi-\frac{1}{2} e^{2 \Phi} \partial_{a} \chi \partial_{b} \chi, \\
0 & =\frac{1}{\sqrt{g_{(5 \mathrm{D})}}} \partial_{a}\left[\sqrt{g_{(5 \mathrm{D})}} g^{a b} \partial_{b} \Phi\right]+e^{2 \Phi} g^{a b} \partial_{a} \chi \partial_{b} \chi, \\
0 & =\frac{1}{\sqrt{g_{(5 \mathrm{D})}}} \partial_{a}\left[\sqrt{g_{(5 \mathrm{D})}} g^{a b} e^{2 \Phi} \partial_{b} \chi\right], \\
\mathcal{R}_{m n} & =-\Lambda g_{m n},
\end{aligned}
$$

where $m, n$ runs from 6 to 9 and $\Lambda=-\frac{6}{R^{2}}$ is the cosmological constant. The last equation is automatically satisfied on a five-sphere, so the 5D effective action could be taken as.

$S_{5 \mathrm{D}}=\frac{1}{2 \kappa_{5}^{2}} \int d^{5} x \sqrt{g_{(5)}}\left[\mathcal{R}^{(5)}-\frac{1}{2}(\partial \Phi)^{2}+\frac{1}{2} e^{2 \Phi}(\partial \chi)^{2}-2 \Lambda\right]$.

The reduction from 10D action to 5D effective action essentially fixes $F_{5}$ and metric components on $S^{5}$. This is justified on the gravity side as we have shown already the ansatz automatically satisfies the field equation. We only turn on 5D metric components, dilaton and axion, all of which have a trivial dependence on $S^{5}$ coordinates. This is sufficient for our purpose, since our goal is to calculate correlators among stress tensor components $O_{E}$ and $\tilde{O}_{E}$ on the field theory side.

\section{B. The fluctuations}

Let us consider the following fluctuations in 5D effective action (3.3):

$$
\begin{aligned}
g_{00} & \rightarrow g_{00}+e^{-i \omega_{E} \tau} r^{2} h_{00}(r), \\
g_{i i} & \rightarrow g_{i i}+e^{-i \omega_{E} \tau} r^{2} h_{i i}(r), \\
\Phi & \rightarrow \Phi+e^{-i \omega_{E} \tau} \delta \Phi(r), \\
\chi & \rightarrow \chi+e^{-i \omega_{E} \tau} \delta \chi(r) .
\end{aligned}
$$

\footnotetext{
${ }^{1}$ Here we have used script $\mathcal{R}$ to denote the curvature in order to distinguish it from the radius $R$ of the bulk.
}

The fluctuations are taken to be a specific Fourier component with Euclidean frequency $\omega_{E}$ and are homogeneous in $\vec{x}$, respecting rotational symmetry. The rotational symmetry also allows us to set $h_{x x}=h_{y y}=h_{z z}=H$. We have imposed the radial gauge, in which $h_{r r}=h_{\mu r}=0$. The equations of motion for the Euclidean fluctuations can be derived as

$$
\begin{aligned}
0= & -16 r^{2} f h_{00}+16 r^{2} f^{2} h_{00}-6 \omega_{E}^{2} f H+2 r^{3} f f^{\prime} h_{00} \\
& +r^{4} f^{\prime 2} h_{00}+12 r^{3} f^{2} h_{00}^{\prime}-r^{4} f f^{\prime} h_{00}^{\prime}+6 r^{3} f^{3} H^{\prime} \\
& +3 r^{4} f^{2} f^{\prime} H^{\prime}+2 r^{4} f^{2} h_{00}^{\prime \prime}, \\
0= & -\omega_{E}^{2} H-8 r^{2} f H+8 r^{2} f^{2} H-r^{3} f^{\prime} h_{00}+2 r^{3} f f^{\prime} H \\
& +r^{3} f h_{00}^{\prime}+8 r^{3} f^{2} H^{\prime}+r^{4} f f^{\prime} H^{\prime}+r^{4} f^{2} H^{\prime \prime}, \\
0= & \frac{3\left(-f^{\prime} H+2 f H^{\prime}\right)}{4 f}+\frac{1}{2} \delta \Phi \Phi^{\prime}-\frac{1}{2} e^{\Phi} \Phi^{\prime} \delta \chi, \\
0= & -2 \delta \Phi\left(\omega_{E}^{2}-2 r^{4} f^{2} \Phi^{\prime 2}\right)-r^{4} h_{00} f^{\prime} \Phi^{\prime}+2 r^{4} f f^{\prime} \delta \Phi^{\prime} \\
& +r^{4} f h_{00}^{\prime} \Phi^{\prime}+10 r^{3} f^{2} \delta \Phi^{\prime}+3 r^{4} f^{2} H^{\prime} \Phi^{\prime} \\
& +4 r^{4} f^{2} e^{\Phi} \delta \chi^{\prime} \Phi^{\prime}+2 r^{4} f^{2} \delta \Phi^{\prime} .
\end{aligned}
$$

We only keep four equations in (3.5). The remaining equations are not independent since they should be satisfied automatically by the solution to the equations in (3.5). Among equations in (3.5), the first two are dynamical equations. The third and fourth equations are constraint equations, which can be combined to eliminate $\delta \chi$, giving rise to a third dynamical equation. As a result, we need to solve only three equations for $h_{00}, H, \delta \Phi$, respectively, by integrating the equations from the horizon. Since the background is Euclidean, we impose a regular boundary condition. Let us consider $\omega_{E}>0$ for now. The solutions take the following series solutions near the horizon:

$$
\begin{aligned}
h_{00}(r)= & B_{0}(r-1)^{\omega_{E} / 4}\left[\frac{-24+12 \omega_{E}}{2+\omega_{E}}(r-1)+\mathcal{O}(r-1)^{2}\right], \\
H(r)= & B_{0}(r-1)^{\omega_{E} / 4} \\
& \times\left[1-\frac{20 \omega_{E}+32 \omega_{E}^{2}+\omega_{E}^{3}}{8\left(2+\omega_{E}\right)^{2}}(r-1)+\mathcal{O}(r-1)^{2}\right], \\
\delta \Phi(r)= & (r-1)^{\omega_{E} / 4}\left[\frac{C_{0}}{-1+q \ln [4(r-1)]}-\frac{6 B_{0}}{\omega_{E}\left(2+\omega_{E}\right)}\right. \\
& \left.-\frac{3\left(\omega_{E}-2\right) B_{0}}{8 q}(-1+q \ln [4(r-1)])+\mathcal{O}(r-1)^{1}\right] .
\end{aligned}
$$

We have set $r_{T}=1$ in the above solution for simplicity. This sets the scale by having $\pi T=1$. The series solutions of $h_{00}$ and $H$ are obtained by solving the first two dynamical equations in (3.5) and we find that there is only one independent solution with normalization constant $B_{0}$. When $\Phi=0$, this is the Euclidean counterpart of the 
infalling solution discussed by Policastro, Son, and Starinets (PSS) [24] in the limit of vanishing spatial momentum. When we have nontrivial $\Phi, \delta \Phi$ satisfies an inhomogeneous dynamical equation whose general solution is the sum of the homogeneous solution (proportional to $B_{0}$ ) and special solution (proportional to $C_{0}$ ). Note that the near horizon solution is modified from a simple series solution due to the nontrivial profile of $\Phi$. Here we organize the solution as a power series in $(r-1)$, treating $\ln (r-1)$ as $\mathcal{O}\left((r-1)^{0}\right)$. (3.6) is essentially all order in $\ln (r-1)$. Integrating these solutions to the boundary, we could obtain two independent solutions with a regularity boundary condition. The number of solutions does not match the number of independent sources. The remaining solutions are pure gauge ones, which do not satisfy the regularity boundary condition [24]. In our case, the pure gauge solution is given by

$h_{\mu \nu}=\frac{1}{r^{2}}\left(\nabla_{\mu} \xi_{\nu}+\nabla_{\nu} \xi_{\mu}\right), \quad \delta \Phi=\xi^{\mu} \partial_{\mu} \Phi, \quad \delta \chi=\xi^{\mu} \partial_{\mu} \chi$.

The gauge function $\xi$ has to be chosen such that fluctuation fields remain in radial gauge. The explicit expressions of the gauge function are found to be

$$
\begin{aligned}
& \xi_{\tau}=e^{-i \omega_{E} \tau} \frac{2\left(r^{4}-1\right) D_{2}-i \omega_{E} D_{1} \sqrt{r^{4}-1}}{2 r^{2}}, \\
& \xi_{r}=e^{-i \omega_{E} \tau} \frac{r}{\sqrt{r^{4}-1}} D_{1} .
\end{aligned}
$$

The corresponding pure gauge solution is given by

$$
\begin{aligned}
h_{00} & =f^{1 / 2}\left(2+\frac{2}{r^{4}}\right) D_{1}-\frac{\omega_{E}^{2} f^{1 / 2}}{r^{2}} D_{1}-2 i \omega_{E} f D_{2}, \\
H & =2 f^{1 / 2} D_{1}, \quad \delta \Phi=\frac{-4 q D_{1}}{r^{4} f^{1 / 2}(1-q \ln f)}, \\
\delta \chi & =\frac{-4 q D_{1}}{r^{4} f^{1 / 2}(1-q \ln f)^{2}} .
\end{aligned}
$$

Notice that there are two independent normalization constants $D_{1,2}$ in (3.8) which precisely generate the remaining two solutions. Hence, we totally have four solutions at hand; they are two numerical ones and two analytic pure gauge solutions. To be specific, let us label the solutions by $i=I, I I, I I I, I V$ with

$$
\begin{aligned}
& I: B_{0}=1, \quad C_{0}=0, \quad D_{1}=D_{2}=0, \\
& I I: B_{0}=0, \quad C_{0}=1, \quad D_{1}=D_{2}=0, \\
& \text { III: } B_{0}=0, \quad C_{0}=0, \quad D_{1}=1, \quad D_{2}=0, \\
& I V: B_{0}=0, \quad C_{0}=0, \quad D_{1}=0, \quad D_{2}=1 \text {. }
\end{aligned}
$$

Then we can analyze the equations of motion near the boundary and calculate the correlation functions among the dual operators from the asymptotic series of the fields $h_{00}$, $H, \delta \Phi$, and $\delta \chi$. We can obtain the following asymptotic behavior:

$$
\begin{aligned}
h_{00} & =a_{0}+\frac{a_{1}}{r^{2}}+\frac{a_{2}}{r^{4}}+\frac{a_{3}}{r^{6}}+\frac{a_{4}}{r^{8}}+\frac{a_{h}}{r^{8}} \ln r+\cdots, \\
H & =b_{0}+\frac{b_{1}}{r^{2}}+\frac{b_{2}}{r^{4}}+\frac{b_{3}}{r^{6}}+\frac{b_{4}}{r^{8}}+\frac{b_{h}}{r^{8}} \ln r+\cdots, \\
\delta \Phi & =f_{0}+\frac{f_{1}}{r^{2}}+\frac{f_{2}}{r^{4}}+\frac{f_{h}}{r^{4}} \ln r+\cdots, \\
\delta \chi & =c_{0}+\frac{c_{1}}{r^{2}}+\frac{c_{2}}{r^{4}}+\frac{c_{h}}{r^{4}} \ln r+\cdots,
\end{aligned}
$$

with the recursion relations among coefficients

$a_{1}=-\frac{b_{0} \omega_{E}^{2}}{2}, \quad a_{2}=-q\left(c_{0}-f_{0}\right)+\frac{3}{2} b_{0}-a_{0}$,

$a_{3}=\frac{\omega_{E}^{2}}{4} b_{0}, \quad a_{h}=\frac{\omega_{E}^{4}}{64}\left(b_{0}+2 b_{2}\right)$,

$b_{1}=0, \quad b_{2}=\frac{q}{3}\left(c_{0}-f_{0}\right)-\frac{1}{2} b_{0}$,

$b_{3}=-\frac{\omega_{E}^{2}}{12}\left(b_{0}+2 b_{2}\right), \quad b_{h}=\frac{\omega_{E}^{4}}{64}\left(b_{0}+2 b_{2}\right)$,

$f_{1}=-\frac{f_{0} \omega_{E}^{2}}{4}, \quad f_{h}=\frac{f_{0} \omega_{E}^{4}}{16}$,

$c_{1}=-\frac{c_{0} \omega_{E}^{2}}{4}, \quad c_{h}=\frac{c_{0} \omega_{E}^{4}}{16}$.

Following the holographic dictionary, we take $a_{0}, b_{0}, c_{0}$, and $f_{0}$ as sources to operators $T_{E}^{00}, T_{E}^{i i}, \tilde{O}_{E}$, and $O_{E}$, respectively. The coefficients $a_{2}, b_{2}, c_{2}$, and $f_{2}$ are corresponding VEVs. It may seems odd that $a_{2}$ and $b_{2}$ are completely determined by recursion relations. We show in Sec. IV B that correlators among $T^{00}$ and $T^{i i}$ are completely fixed by Ward identities in the limit of vanishing spatial momentum, making $a_{2}$ and $b_{2}$ nondynamical. The coefficients $c_{2}, f_{2}$ cannot be determined by analyzing the boundary behavior only. They are related through the constraint equation,

$$
c_{2}=\frac{-6 b_{2}+24 b_{4}-3 b_{h}+4 q f_{2}-4 q^{2} f_{0}}{4 q} .
$$

The coefficients $a_{4}$ and $b_{4}$ are not determined up to the order we work. In order to calculate the correlation function, we turn on the sources for the operators and measure the corresponding VEVs. The ratio of the VEV to the sources can be defined by the following response matrix: 


$$
\left(\begin{array}{cccc}
G_{a a} & G_{a b} & G_{a c} & G_{a f} \\
G_{b a} & G_{b b} & G_{b c} & G_{b f} \\
G_{c a} & G_{c b} & G_{c c} & G_{c f} \\
G_{f a} & G_{f b} & G_{f c} & G_{f f}
\end{array}\right)=\left(\begin{array}{llll}
\frac{\partial a_{2}}{\partial a_{0}} & \frac{\partial a_{2}}{\partial b_{0}} & \frac{\partial a_{2}}{\partial c_{0}} & \frac{\partial a_{2}}{\partial f_{0}} \\
\frac{\partial b_{2}}{\partial a_{0}} & \frac{\partial b_{2}}{\partial b_{0}} & \frac{\partial b_{2}}{\partial c_{0}} & \frac{\partial b_{2}}{\partial f_{0}} \\
\frac{\partial c_{2}}{\partial a_{0}} & \frac{\partial c_{2}}{\partial b_{0}} & \frac{\partial c_{2}}{\partial c_{0}} & \frac{\partial c_{2}}{\partial f_{0}} \\
\frac{\partial f_{2}}{\partial a_{0}} & \frac{\partial f_{2}}{\partial b_{0}} & \frac{\partial f_{2}}{\partial c_{0}} & \frac{\partial f_{2}}{\partial f_{0}}
\end{array}\right) .
$$

Because of operator mixing in the renormalization group flow, the off-diagonal matrix elements in the response matrix is nonvanishing. For any given basis solution, the response matrix satisfies

$$
\left(\begin{array}{c}
a_{2}^{i} \\
b_{2}^{i} \\
c_{2}^{i} \\
f_{2}^{i}
\end{array}\right)=\left(\begin{array}{cccc}
G_{a a} & G_{a b} & G_{a c} & G_{a f} \\
G_{b a} & G_{b b} & G_{b c} & G_{b f} \\
G_{c a} & G_{c b} & G_{c c} & G_{c f} \\
G_{f a} & G_{f b} & G_{f c} & G_{f f}
\end{array}\right)\left(\begin{array}{c}
a_{0}^{i} \\
b_{0}^{i} \\
c_{0}^{i} \\
f_{0}^{i}
\end{array}\right)
$$

for $i=I, I I, I I I, I V$. Since we have in total four basis solutions, we can use them to calculate the response matrix efficiently as

$$
\begin{gathered}
\left(\begin{array}{cccc}
G_{a a} & G_{a b} & G_{a c} & G_{a f} \\
G_{b a} & G_{b b} & G_{b c} & G_{b f} \\
G_{c a} & G_{c b} & G_{c c} & G_{c f} \\
G_{f a} & G_{f b} & G_{f c} & G_{f f}
\end{array}\right) \\
=\left(\begin{array}{cccc}
a_{2}^{I} & a_{2}^{I I} & a_{2}^{I I I} & a_{2}^{I V} \\
b_{2}^{I} & b_{2}^{I I} & b_{2}^{I I I} & b_{2}^{I V} \\
c_{2}^{I} & c_{2}^{I I} & c_{2}^{I I I} & c_{2}^{I V} \\
f_{2}^{I} & f_{2}^{I I} & f_{2}^{I I I} & f_{2}^{I V}
\end{array}\right)\left(\begin{array}{cccc}
a_{0}^{I} & a_{0}^{I I} & a_{0}^{I I I} & a_{0}^{I V} \\
b_{0}^{I} & b_{0}^{I I} & b_{0}^{I I I} & b_{0}^{I V} \\
c_{0}^{I} & c_{0}^{I I} & c_{0}^{I I I} & c_{0}^{I V} \\
f_{0}^{I} & f_{0}^{I I} & f_{0}^{I I I} & f_{0}^{I V}
\end{array}\right)^{-1}
\end{gathered}
$$

Most of the matrix elements on the rhs of (3.16) are known analytically. From solutions $I I, I I I$, and $I V$, we easily obtain

$a_{0}^{I I}=0, \quad b_{0}^{I I}=0, \quad a_{2}^{I I}=0, \quad b_{2}^{I I}=0, \quad b_{4}^{I I}=0$,

$a_{0}^{I I I}=2, \quad b_{0}^{I I I}=2, \quad f_{0}^{I I I}=0, \quad a_{2}^{I I I}=1$,

$b_{2}^{I I I}=-1, \quad f_{2}^{I I I}=-4 q, \quad b_{4}^{I I I}=-\frac{1}{4}$,

$a_{0}^{I V}=-2 i \omega, \quad b_{0}^{I V}=0, \quad f_{0}^{I V}=0$,

$a_{2}^{I V}=2 i \omega, \quad b_{2}^{I V}=0, \quad f_{2}^{I V}=0, \quad b_{4}^{I V}=0$.

With recursion relations (3.12) and constraint (3.13), we can fix most entries of the response matrix as

$$
\left(\begin{array}{cccc}
G_{a a} & G_{a b} & G_{a c} & G_{a f} \\
G_{b a} & G_{b b} & G_{b c} & G_{b f} \\
G_{c a} & G_{c b} & G_{c c} & G_{c f} \\
G_{f a} & G_{f b} & G_{f c} & G_{f f}
\end{array}\right)=\left(\begin{array}{cccc}
-1 & 3 / 2 & -q & q \\
0 & -1 / 2 & q / 3 & -q / 3 \\
0 & -2 q & \times & \times \\
0 & -2 q & \times & \times
\end{array}\right) .
$$

The entries marked with " $x$ " represent the value which has to be determined numerically. In fact, the four undetermined entries are not all independent. We can show that there is a less obvious identity among them

$$
G_{c f}-G_{f c}+2 q=G_{f f}-G_{c c} .
$$

Finally we remark on one important property of the response matrix under the sign flip of $\omega_{E}$. Up to now we have considered $\omega_{E}>0$; the situation with $\omega_{E}<0$ is easy to analyze: the regularity condition requires the solutions near horizon $\sim(r-1)^{-\omega_{E} / 4}$. It follows that solutions $I$ and $I I$ remain unchanged under the sign flip. On the other hand, the explicit pure gauge solutions show that $I I I$ is also unchanged, while $I V$ changes sign. Combining all these with recursion relations (3.12) and constraint (3.13), we can show that the response matrix is also unchanged under the sign flip of $\omega_{E}$. This will be useful in the following.

\section{EUCLIDEAN AND RETARDED CORRELATORS}

\section{A. Euclidean correlators}

The D3-brane-D-instanton background is found in Euclidean spacetime with nontrivial profiles of dilaton and axion which is consistent with the field theory expectation since the instanton only exists in Euclidean field theory. The effect of the instanton to real world physics is usually understood as a tunneling process. We will calculate correlators among stress tensor, glueball, and topological charge density in this section, which are dual to the perturbation of the metric, dilaton and axion, respectively, as discussed in the previous sections. Notice that the resulting correlators are all Euclidean in our current analysis of the bulk gravity. In the next section, we will study the counterpart in Minkowskian spacetime.

We show in the Appendix how to obtain correlators among $T_{E}^{00}, T_{E}^{i i}, O_{E}, \tilde{O}_{E}$ from (3.18) and (A5). Here, the sum over $i$ is assumed in $T_{E}^{i i}$ as this is the quantity coupled to the isotropic metric perturbation $H(r \rightarrow \infty)$. Respectively, the correlators are given by 


$$
\begin{aligned}
G_{00,00}^{E} & =\int d \tau d^{3} x e^{i \omega_{E} \tau}\left\langle T_{E}^{00}(\tau, x) T_{E}^{00}(0)\right\rangle=3, \\
G_{00, i i}^{E} & =\int d \tau d^{3} x e^{i \omega_{E} \tau}\left\langle T_{E}^{00}(\tau, x) T_{E}^{i i}(0)\right\rangle=3, \\
G_{i i, j j}^{E} & =\int d \tau d^{3} x e^{i \omega_{E} \tau}\left\langle T_{E}^{i i}(\tau, x) T_{E}^{j j}(0)\right\rangle=-9, \\
G_{00, O}^{E} & =\int d \tau d^{3} x e^{i \omega_{E} \tau}\left\langle T_{E}^{00}(\tau, x) O_{E}(0)\right\rangle=4 q, \\
G_{00, \tilde{O}}^{E} & =\int d \tau d^{3} x e^{i \omega_{E} \tau}\left\langle T_{E}^{00}(\tau, x) \tilde{O}_{E}(0)\right\rangle=-4 q, \\
G_{i i, O}^{E} & =\int d \tau d^{3} x e^{i \omega_{E} \tau}\left\langle T_{E}^{i i}(\tau, x) O_{E}(0)\right\rangle=-4 q, \\
G_{i i, \tilde{O}}^{E} & =\int d \tau d^{3} x e^{i \omega_{E} \tau}\left\langle T_{E}^{i i}(\tau, x) \tilde{O}_{E}(0)\right\rangle=4 q, \\
G_{O O}^{E} & =\int d \tau d^{3} x e^{i \omega_{E} \tau}\left\langle O_{E}(\tau, x) O_{E}(0)\right\rangle=4 G_{f f}, \\
G_{\tilde{O} \tilde{O}}^{E} & =\int d \tau d^{3} x e^{i \omega_{E} \tau}\left\langle\tilde{O}_{E}(\tau, x) \tilde{O}_{E}(0)\right\rangle=-4 G_{c c}, \\
G_{O \tilde{O}}^{E} & =\int d \tau d^{3} x e^{i \omega_{E} \tau}\left\langle O_{E}(\tau, x) \tilde{O}_{E}(0)\right\rangle \\
& =-2 G_{c f}+2 G_{f c}-4 q .
\end{aligned}
$$

$$
\int d \tau d^{3} x e^{i \omega_{E} \tau}\left\langle\tilde{O}_{E}(\tau, x) T_{E}^{00}(0)\right\rangle=-4 q
$$

A similar procedure with $\delta g_{00}=0$ and $\delta g_{i i}=H(r \rightarrow \infty)$ leads to the correlator:

$$
\int d \tau d^{3} x e^{i \omega_{E} \tau}\left\langle\tilde{O}_{E}(\tau, x) T_{E}^{i i}(0)\right\rangle=4 q
$$

It is not difficult to see that they are equivalent to the corresponding correlators in (4.1) upon using complex conjugation.

Furthermore, we can obtain from (3.19) the following relation among Euclidean correlators:

$$
G_{O \tilde{O}}^{E}+2 q+\frac{1}{2}\left(G_{O O}^{E}-G_{\tilde{O} \tilde{O}}^{E}\right)=0
$$

\section{B. Ward identities}

In this section, we will show that the apparently trivial form of the correlators are in fact a consequence of Ward identities following from diffeomorphism invariance and conformal invariance of the action:

$$
\begin{aligned}
S\left[g_{\mu \nu}^{4 \mathrm{D}}, \phi^{4 \mathrm{D}}, \chi^{4 \mathrm{D}}\right]= & S\left[g_{\mu \nu}^{4 \mathrm{D}}+\nabla_{\mu} \xi_{\nu}^{4 \mathrm{D}}+\nabla_{\nu} \xi_{\mu}^{4 \mathrm{D}}, \phi^{4 \mathrm{D}}\right. \\
& \left.+\xi^{\mu} \partial_{\mu} \phi^{4 \mathrm{D}}, \chi^{4 \mathrm{D}}+\xi^{\mu} \partial_{\mu} \chi^{4 \mathrm{D}}\right] \\
S\left[g_{\mu \nu}^{4 \mathrm{D}}, \phi^{4 \mathrm{D}}, \chi^{4 \mathrm{D}}\right]= & S\left[\Omega^{2} g_{\mu \nu}^{4 \mathrm{D}}, \phi^{4 \mathrm{D}}, \chi^{4 \mathrm{D}}\right] .
\end{aligned}
$$

We have used the label "4D" to indicate that all the variables in (4.7a) are four-dimensional, i.e., they are sources corresponding to $a_{0}, b_{0}, c_{0}, f_{0}$. Varying (4.7a) with respect to $\xi_{\mu}^{4 \mathrm{D}}(x)$ and $(4.7 \mathrm{~b})$ with respect to $\Omega$, we obtain

$$
\begin{aligned}
\delta \int d \tau d^{3} x \sqrt{g} \tilde{O}_{E}(x) & =\int d \tau d^{3} x \sqrt{g}\left[\frac{1}{2} g^{\mu \nu} \delta g_{\mu \nu} \tilde{O}_{E}(x)+\delta \tilde{O}_{E}\right] \\
& =0
\end{aligned}
$$$$
-\nabla_{\mu} T_{E}^{\mu \nu}(x)+O_{E}(x) \nabla^{\nu} \phi^{4 \mathrm{D}}(x)+\tilde{O}_{E}(x) \nabla^{\nu} \chi^{4 \mathrm{D}}(x)=0,
$$

$$
g_{\mu \nu}\left\langle T^{\mu \nu}\right\rangle=0
$$

Varying (4.8b) with respect to $g_{\lambda \rho}$ and setting $g_{\mu \nu}=\delta_{\mu \nu}$, $\phi^{4 \mathrm{D}}=\chi^{4 \mathrm{D}}=0$, we obtain

$$
\frac{\delta\left\langle\tilde{O}_{E}(x)\right\rangle}{\delta g_{00}(y)}=-2 q \delta^{4}(x-y)
$$

What we obtain above is nothing but the correlator $\left\langle\tilde{O}_{E}(x) T_{E}^{00}(y)\right\rangle$. It takes a familiar form in momentum space $^{2}$

\footnotetext{
${ }^{2}$ The coupling $\int d^{4} x \frac{1}{2} h_{\mu \nu} T^{\mu \nu}$ brings in an additional factor
of 2 .
}

$q_{\mu}\left(G_{E}^{\mu \nu, \lambda \rho}(q)+\delta^{\nu \lambda}\left\langle T_{E}^{\mu \rho}\right\rangle+\delta^{\nu \rho}\left\langle T_{E}^{\mu \lambda}\right\rangle-\delta^{\mu \nu}\left\langle T_{E}^{\lambda \rho}\right\rangle\right)$
$\delta_{\mu \nu} G_{E}^{\mu \nu, \lambda \rho}=-2\left\langle T_{E}^{\lambda \rho}\right\rangle$

The derivation is parallel to the one in [24] except that our background metric is flat Euclidean. We can show that, in fact, (4.9) alone is enough to fix correlators $G_{E}^{00,00}, G_{E}^{00, i i}$, and $G_{E}^{i i, j j}$. Note that $q_{\mu}=\left(\omega_{E}, \mathbf{0}\right)$. We obtain from (4.9) 


$$
\begin{aligned}
\omega_{E}\left(G_{E}^{00,00}+\left\langle T_{E}^{00}\right\rangle\right) & =0, \\
\omega_{E}\left(G_{E}^{00, i i}-\left\langle T_{E}^{i i}\right\rangle\right) & =0, \\
G_{E}^{00, j j}+G_{E}^{i i, j j} & =-2\left\langle T_{E}^{i i}\right\rangle,
\end{aligned}
$$

where we always assume summation over repeated indices. Using $\left\langle T_{E}^{00}\right\rangle=-\left\langle T_{E}^{i i}\right\rangle=-3 P$, we obtain

$$
G_{E}^{00,00}=3 P, \quad G_{E}^{00, i i}=3 P, \quad G_{E}^{i i, j j}=-9 P .
$$

It indeed agrees with (4.1) and explains the nondynamical origin of the correlators.

Now we derive a new set of Ward identities involving $O_{E}$ and $\tilde{O}_{E}$. Varying $(4.8 \mathrm{~b})$ with respect to $\phi^{4 \mathrm{D}}(y)$ and $\chi^{4 \mathrm{D}}(y)$ and setting $g_{\mu \nu}=\delta_{\mu \nu}, \phi^{4 \mathrm{D}}=\chi^{4 \mathrm{D}}=0$, we obtain from (4.7a):

$$
-q_{\mu} G_{E}^{\mu \nu, O}-q^{\nu}\left\langle O_{E}\right\rangle=0, \quad-q_{\mu} G_{E}^{\mu \nu, \tilde{O}}-q^{\nu}\left\langle\tilde{O}_{E}\right\rangle=0 .
$$

Note that $\left\langle O_{E}(x)\right\rangle=-\left\langle\tilde{O}_{E}(x)\right\rangle=-4 q$, we obtain immediately

$$
G_{E}^{00, O}=4 q \quad G_{E}^{00, \tilde{O}}=-4 q .
$$

Similarly, we can obtain from (4.7b)

$$
\begin{gathered}
\delta_{\mu \nu}(x)\left\langle T_{E}^{\mu \nu}(x) O_{E}(0)\right\rangle=0, \\
\delta_{\mu \nu}(x)\left\langle T_{E}^{\mu \nu}(x) \tilde{O}_{E}(0)\right\rangle=0 .
\end{gathered}
$$

The Fourier transform of (4.14) gives

$$
G_{E}^{00, O}+G_{E}^{i i, O}=0 \quad G_{E}^{00, \tilde{O}}+G_{E}^{i i, \tilde{O}}=0 .
$$

Combining with (4.13), we obtain

$$
G_{E}^{i i, O}=-4 q \quad G_{E}^{i i, \tilde{O}}=4 q .
$$

All the cross correlators are fixed by Ward identities in the limit of vanishing spatial momentum.

\section{Analytic continuation}

To see how the presence of an instanton affects real time dynamics, we need to know the corresponding correlators in Minkowski spacetime. The conventional holographic approach is to directly work with Minkowski background, which automatically gives a real time correlator. However this is not applicable to the D-instanton background: A naive Wick rotation of the background would lead to purely imaginary VEVs for $O$ and $\tilde{O}$. This is, in fact, consistent with the field theory expectation that the instanton exists only in Euclidean space. So only the Euclidean correlator is well defined. Nevertheless, an analytic continuation between the Euclidean correlator and the real time correlator is possible in field theory. We will use the following identity between the retarded correlator and the Euclidean correlator:

$$
G^{R}\left(i \omega_{E}\right)=-G^{E}\left(\omega_{E}\right)
$$

where $\omega_{E}$ takes values of the Matsubara frequency $\omega_{E}=2 \pi T n$. A clean derivation of (4.17) can be found, for example, in [25]. In (4.17), $G^{R}$ and $G^{E}$ are defined with the same operator. It applies to the case of dilaton, whose explicit Euclidean and Minkowskian correlators are given by

$$
\begin{aligned}
G_{O O}^{R}(\omega) & =-i \int d t d^{3} x e^{i \omega t}\langle[O(t, x), O(0)]\rangle, \\
G_{O O}^{E}\left(\omega_{E}\right) & =\int d \tau d^{3} x e^{i \omega_{E} \tau}\left\langle O_{E}(\tau, x) O_{E}(0)\right\rangle,
\end{aligned}
$$

with $O=O_{E}$. The Minkowskian and Euclidean times are related by $i t=\tau$, correspondingly $\omega=i \omega_{E}$. We have already evaluated the Euclidean correlators in the D-instanton background. Through (4.17) they give us automatically the retarded correlators, albeit defined only on $\omega=i 2 \pi T n$. To extend the results to real frequency, we need to analytically continue (4.17). There is a natural continuation:

$$
G_{O O}^{R}(\omega)=-G_{O O}^{E}(-i \omega)
$$

with $\omega$ being real. (4.19) indicates that to obtain the retarded correlator with real $\omega$, we need to evaluate the Euclidean correlator with purely imaginary frequency $-i \omega$. Note that we have been working with $\omega_{E}>0$ for the evaluation of Euclidean correlators. The horizon solution fixed by the regularity condition behaves as $\sim(r-1)^{\omega_{E} / 4}$. The horizon solution can be extended to the right half plane $R e \omega_{E} \geq 0$. Using the relation $\omega_{E}=-i \omega$, it is mapped to the upper half plane of $\omega$. Note that the horizon solution in terms of $\omega$ is precisely an infalling wave type $\sim(r-1)^{-i \omega / 4}$. Had we started by extending the left half plane of $\omega_{E}$, where the horizon solution behaves as $\sim(r-1)^{-\omega_{E} / 4}$, we would not obtain the infalling horizon solution by the same mapping $\omega_{E}=-i \omega$. To further confirm the prescription, we show that the analytic continuation (4.19) holds for known examples of super-Yang-Mills plasma with $q=0$ where the direct calculation of both Euclidean and retarded correlators is possible. In this case, the EOM of dilaton decouples from the metric perturbation. It is given by

$-2 \omega_{E}^{2} \delta \Phi+r^{3}\left(2 r f f^{\prime} \delta \Phi^{\prime}+f^{2}\left(10 \delta \Phi^{\prime}+2 r \delta \Phi^{\prime \prime}\right)\right)=0$.

The EOM of dilaton in the Minkowski background is given by the mapping $\omega_{E}=-i \omega$ :

$2 \omega^{2} \delta \Phi+r^{3}\left(2 r f f^{\prime} \delta \Phi^{\prime}+f^{2}\left(10 \delta \Phi^{\prime}+2 r \delta \Phi^{\prime \prime}\right)\right)=0$. 
Note that the regular horizon solution in the Euclidean case is also mapped to the infalling horizon solution in the Minkowskian case. Taking into account the convention in the definition (4.18), we readily confirm the equality (4.19) for $\operatorname{Re}_{E} \geq 0$.

Next, we discuss autocorrelator of $\tilde{O}$ defined as

$$
\begin{aligned}
G_{\tilde{O} \tilde{o}}^{R}(\omega) & =-i \int d t d^{3} x e^{i \omega t}\langle[\tilde{O}(t, x), \tilde{O}(0)]\rangle, \\
G_{\tilde{O} \tilde{O}}^{E}\left(\omega_{E}\right) & =\int d \tau d^{3} x e^{i \omega_{E} \tau}\left\langle\tilde{O}_{E}(\tau, x) \tilde{O}_{E}(0)\right\rangle .
\end{aligned}
$$

In contrast to the above case, $\tilde{O}$ and $\tilde{O}_{E}$ differ by a factor. To see this, we write down the field theory definitions for $\tilde{O}$ and $\tilde{O}_{E}$ (for comparison we also include $O$ and $O_{E}$ ):

$O_{E}=\operatorname{tr} \mathcal{F}_{E}^{2}=\operatorname{tr} \mathcal{E}_{E}^{2}+\operatorname{tr} \mathcal{B}_{E}^{2}, \quad O=\operatorname{tr} \mathcal{F}^{2}=-\operatorname{tr} \mathcal{E}^{2}+\operatorname{tr} \mathcal{B}^{2}$,

$\tilde{O}_{E}=\operatorname{tr} \mathcal{F}_{E} \wedge \mathcal{F}_{E}=\operatorname{tr} \mathcal{E}_{E} \cdot \mathcal{B}_{E}, \quad \tilde{O}=\operatorname{tr} \mathcal{F} \wedge \mathcal{F}=\operatorname{tr} \mathcal{E} \cdot \mathcal{B}$,

with $\mathcal{E}$ and $\mathcal{B}$ being chromo electric and magnetic fields. Note that $i t=\tau$. We obtain $\partial_{t}=i \partial_{\tau}$, thus $\mathcal{E}_{E}=-i \mathcal{E}$ and $\mathcal{B}_{E}=\mathcal{B}$. It is then straightforward to verify

$$
O_{E}=O, \quad \tilde{O}_{E}=i \tilde{O}
$$

Consequently, the analytic continuation for the autocorrelator of $\tilde{O}$ involves an additional minus sign.

$$
G_{\tilde{o} \tilde{o}}^{R}(\omega)=G_{\tilde{O} \tilde{o}}^{E}(-i \omega) .
$$

We can confirm the prescription at $q=0$ by the following indirect comparison. Note that retarded correlators for $O$ and $\tilde{O}$ are degenerate in the Minkowskian D3-brane background because the dual dilaton and axion satisfy the same equation of motion (EOM) [26]. Therefore we would obtain from (4.25)

$$
G_{\tilde{o} \tilde{o}}^{E}(-i \omega)=G_{\tilde{o} \tilde{O}}^{R}(\omega)=G_{O O}^{R}(\omega)=-G_{O O}^{E}(-i \omega) .
$$

Below we confirm (4.26), where both sides can be calculated with (4.1). Note that axion and dilaton also satisfy the same EOM in the Euclidean background, which simply gives $G_{f f}=G_{c c}$. However, the kinetic terms of dilaton and axion differ in sign. The sign difference is reflected in the equations of $G_{O O}^{E}$ and $G_{\tilde{O} \tilde{O}}^{E}$ in (4.1). Thus, we find $G_{\tilde{O} \tilde{o}}^{E}(-i \omega)=-G_{O O}^{E}(-i \omega)$, consistent with (4.25).

Finally, we discuss the remaining correlators among stress tensor components. Note that, under Wick rotation, the Euclidean and Minkowskian operators are related by

$$
T_{E}^{00}=-T^{00}, \quad T_{E}^{i i}=T^{i i} .
$$

It follows that the analytic continuation is modified to

$$
\begin{aligned}
G_{R}^{00,00}(\omega) & =-G_{E}^{00,00}(-i \omega), \\
G_{R}^{00, i i}(\omega) & =G_{E}^{00, i i}(-i \omega), \\
G_{R}^{i i, j j}(\omega) & =-G_{E}^{i i, j j}(-i \omega) .
\end{aligned}
$$

Since we already have explicit results for the Euclidean correlator among stress tensor components, we can make a direct comparison with their Minkowskian counterpart in PSS [24]. Setting the spatial momentum to zero in their case and noting that our $G^{R}$ corresponds to their $G$, we readily confirm the correctness of the analytic continuation (4.28).

To proceed with the case $q \neq 0$, we simply apply the above prescription: we numerically integrate the horizon solution (3.6) with $\omega_{E}=-i \omega$ for $\omega>0$ to the boundary and match to asymptotic series (3.11) to determine the response matrix using (3.16). With elements of the response matrix, we can use (4.1) to determine the boundary correlator.

\section{Results on retarded correlators}

Now we are ready to use (4.25) to study retarded correlators for $O$ and $\tilde{O}$, respectively. The retarded correlators are readily obtained from (4.1) as

$$
\begin{gathered}
G_{O O}^{R}(\omega)=-4 G_{f f}(-i \omega), \\
G_{\tilde{O} \tilde{o}}^{R}(\omega)=-4 G_{c c}(-i \omega) .
\end{gathered}
$$

The results are known for the case $q=0$, where $G_{O O}^{R}$ and $G_{\tilde{o} \tilde{o}}^{R}$ are degenerate. Our numerical results indicate the degeneracy is still true when $q \ll \omega, q \ll T$. Furthermore, in the regime $\omega \ll T$, the correlators display diffusive behavior as

$$
G_{O O}^{R}=G_{\tilde{o} \tilde{O}}^{R}=-i \omega \Gamma_{\mathrm{CS}} / T,
$$

where $\Gamma_{\mathrm{CS}}$ is the diffusion constant of the Chern-Simons (CS) number [26].

We are interested in the regime $q \sim O(T)$. This is where the instanton effect becomes significant. We first look at the regime $\omega \ll T$. In Fig. 1 we show the $\omega$ and $q$ dependencies of the real and imaginary parts of $G_{O O}^{R}$ and $-G_{\tilde{O}}^{R} \tilde{o}$. We find the imaginary parts of $G_{O O}^{R}$ and $-G_{\tilde{O} \tilde{O}}^{R}$ are almost indistinguishable. Note that this is in contrast to the degeneracy of $G_{O O}^{R}$ and $G_{\tilde{O} \tilde{O}}^{R}$ (without minus sign) in the regime $q \ll T$. The numerical results also indicate the following scaling relation:

$\operatorname{Re} G_{O O}^{R} \sim q^{2}, \quad \operatorname{Im} G_{O O}^{R} \simeq-\operatorname{Im} G_{\tilde{O} \tilde{O}}^{R} \sim q^{2} / \omega$.

We further take a closer look at the $q$ dependence of $G_{O O}^{R}+G_{\tilde{O} \tilde{O}}^{R}$ in Fig. 2. For the real part, Fig. 2 shows that 

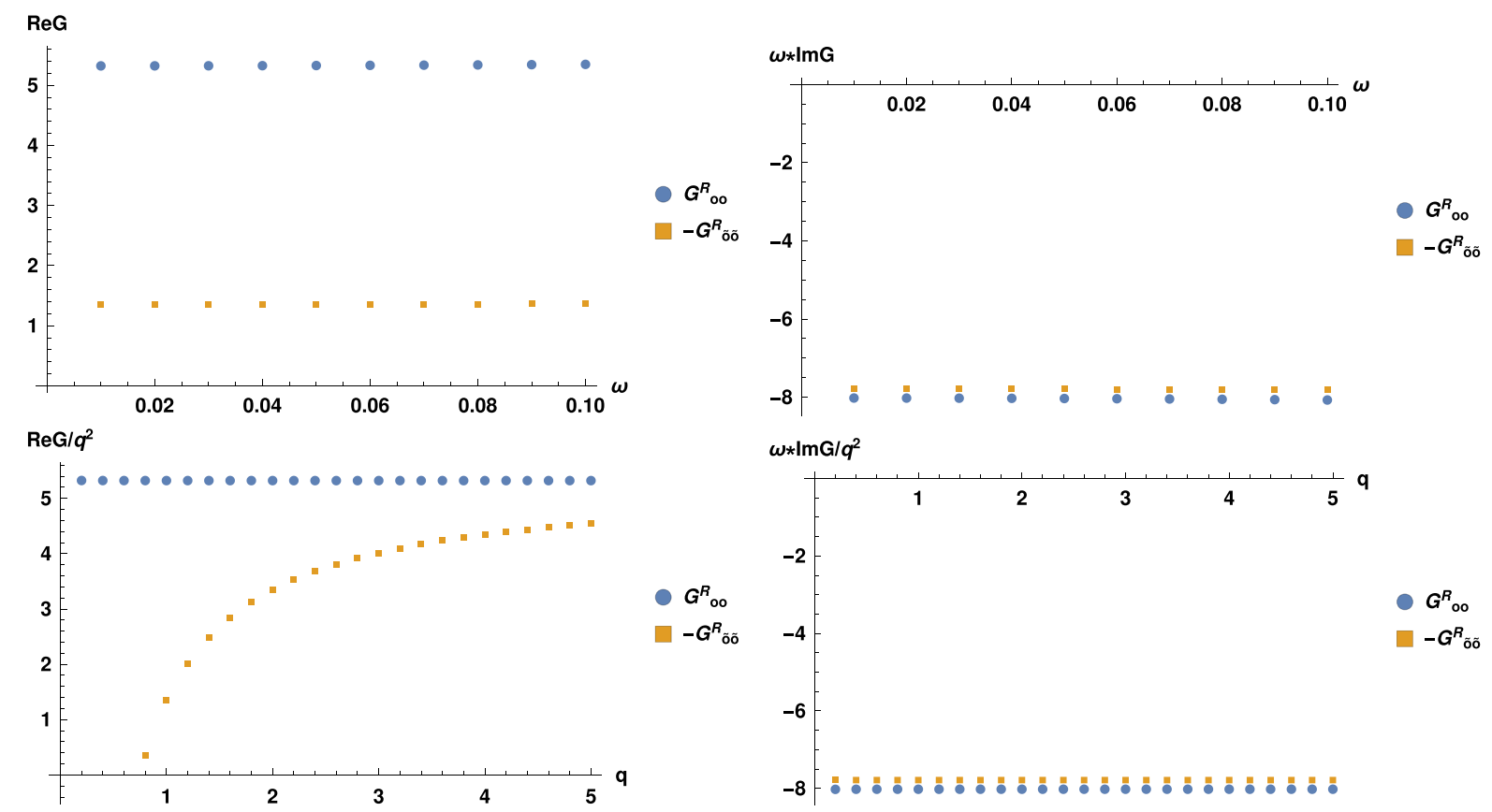

FIG. 1. The top panels show the real and imaginary parts of $G_{O O}^{R}$ and $-G_{\tilde{O} \tilde{O}}^{R}$ in units of $P$ at $q /(\pi T)^{4}=1$ as a function of $\omega$. In the low frequency regime, the real parts of $G_{O O}^{R}$ and $-G_{\tilde{O} \tilde{O}}^{R}$ differ and are almost independent of $\omega$. On the other hand, the imaginary parts of $G_{O O}^{R}$ and $G_{\tilde{O} \tilde{O}}^{R}$ are almost indistinguishable. We use an offset to guide the eyes. The numerical results indicate $\operatorname{Im} G_{O O}^{R} \simeq \operatorname{Im} G_{\tilde{O} \tilde{O}}^{R} \sim 1 / \omega$. The bottom panels show the $q$ dependence of the real and imaginary parts of $G_{O O}^{R}$ and $-G_{\tilde{O} \tilde{O}}^{R}$ in units of $P$ at $\omega /(\pi T)=0.01$. The numerical results indicate $\operatorname{Re} G_{O O}^{R} \sim q^{2}$ and $\operatorname{Im} G_{O O}^{R} \simeq \operatorname{Im} G_{\tilde{O} \tilde{O}}^{R} \sim q^{2}$. The $q^{2}$ dependence extends to a rather large value of $q$.
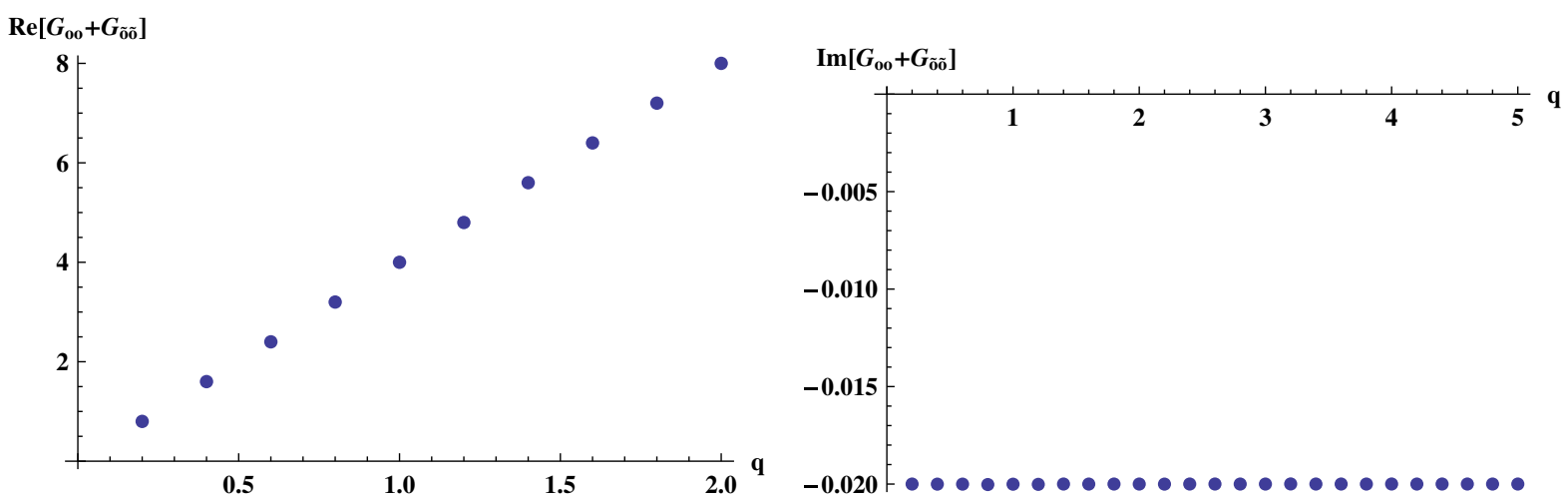

FIG. 2. The real and imaginary part of $G_{O O}^{R}+G_{\tilde{O} \tilde{O}}^{R}$ in units of $P$ as a function of $q$ at $\omega /(\pi T)=0.01$. The real part shows a clear linear dependence, while the imaginary part is almost $q$ independent.

the difference of $\operatorname{Re} G_{O O}^{R}$ and $-\operatorname{Re} G_{\tilde{O} \tilde{O}}^{R}$ as seen in Fig 1 is linear in $q$, suggesting the following scaling behavior: $\operatorname{Re} G_{\tilde{O} \tilde{O}}^{R} \sim q^{2}+\# q$. For the imaginary part, Fig. 2 shows there is, in fact, a small difference not visible in Fig. 1.

Moving on from the regime $\omega \ll T$, we study a wider range of $\omega$. We expect when $\omega \gg q$, the instanton effect becomes negligible; thus, $G_{O O}^{R}$ and $G_{\tilde{O} \tilde{O}}^{R}$ again become degenerate. We show the $\omega$ and $q$ dependence of $G_{O O}^{R}$ and $G_{\tilde{O} \tilde{O}}^{R}$ in a wider range of $\omega$. The degeneracy of $G_{O O}^{R}$ and
$-G_{\tilde{O} \tilde{O}}^{R}$ in the small $\omega$ regime and the degeneracy $G_{O O}^{R}$ and $G_{\tilde{O} \tilde{O}}^{R}$ in the large $\omega$ regime are visible in Fig. 3. The figure also suggests the saturation of $q^{2}$ dependence for both real and imaginary parts of $G_{O O}^{R}$ and $G_{\tilde{O} \tilde{O}}^{R}$.

\section{DISCUSSION}

The regime with $\omega \ll T$ and $q \sim O\left(T^{4}\right)$ deserves special attention. Numerical results suggest the scaling (4.31), which after reinstating the dimension gives 


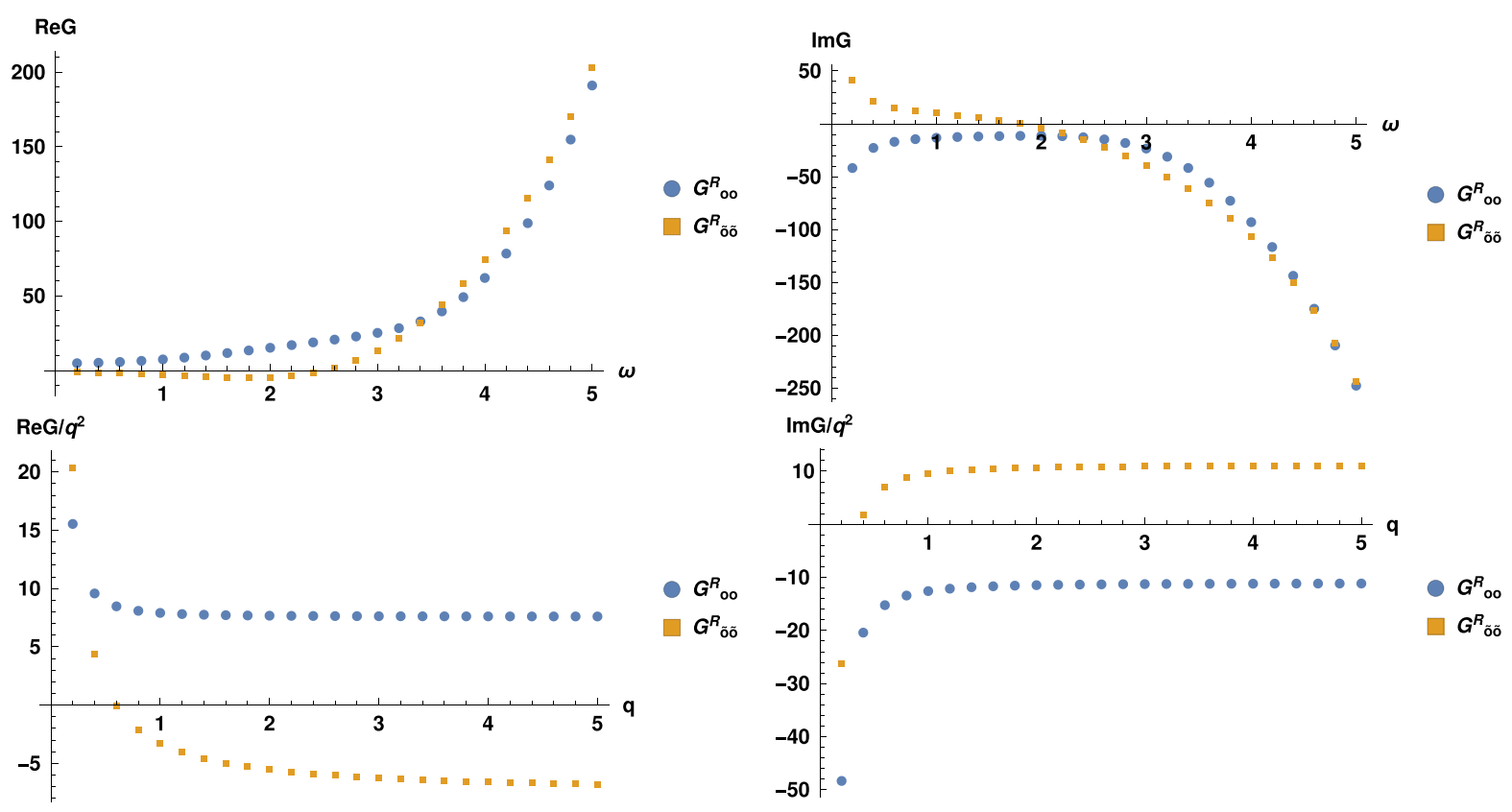

FIG. 3. The top panels show the real and imaginary parts of $G_{O O}^{R}$ and $G_{\tilde{O} \tilde{o}}^{R}$ in units of $P$ at $q /(\pi T)^{4}=1$ as a function of $\omega$. At small $\omega$, we recover the degeneracy of $G_{O O}^{R}$ and $-G_{\tilde{o} \tilde{o}}^{R}$ found before. At large $\omega$, the plot reveals the degeneracy of $G_{O O}^{R}$ and $G_{\tilde{O} \tilde{O}}^{R}$ instead as expected on general ground. The bottom panels show the $q$ dependence of the real and imaginary parts of $G_{O O}^{R}$ and $G_{\tilde{O} \tilde{O}}^{R}$ in units of $P$ at $\omega /(\pi T)=1$. At large $q$, numerical results suggest a $q^{2}$ dependence. Note that this regime is close to the degeneracy regime of $G_{O O}^{R}$ and $-G_{\tilde{O} \tilde{O}}^{R}$.

$$
\operatorname{Im} G_{O O}^{R}(\omega) \simeq-\operatorname{Im} G_{\tilde{O} \tilde{o}}^{R}(\omega)=-\frac{q^{2} \Gamma}{\omega}
$$

where $\Gamma \sim O\left(\frac{1}{T^{3}}\right)$. From Fig 1 we can see $\Gamma$ is positive. Note that the imaginary part of the retarded correlator is related to the spectral density $\chi_{O}$ and $\chi_{\tilde{O}}$ as

$$
\chi_{O}=-2 \operatorname{Im} G_{O O}^{R}, \quad \chi_{\tilde{O}}=-2 \operatorname{Im} G_{\tilde{O} \tilde{o}}^{R} .
$$

The spectral density should be positive for positive frequency $\omega$ [27]. Combining with (5.1), we find indeed positive spectral density for $O$, but negative spectral density for $\tilde{O}$. Away from the regime, we do recover positive spectral density for both $O$ and $\tilde{O}$ from Fig. 3. We suggest the violation of the positive condition for $\tilde{O}$ in the regime is related to the existence of the unstable fluctuation of $\tilde{O}$. To see this, we consider the following correlator:

$$
\int d^{3} x\left\langle(\tilde{O}(t, x)-\tilde{O}(0))^{2}\right\rangle
$$

Using the Kubo-Martin-Schwinger relation, we can express (5.3) in terms of the imaginary part of the retarded correlator

$$
\begin{aligned}
\int & d^{3} x\left\langle(\tilde{O}(t, x)-\tilde{O}(0))^{2}\right\rangle \\
& =\int \frac{d \omega}{2 \pi}\left(2-e^{-i \omega t}-e^{i \omega t}\right) G_{\tilde{O} \tilde{O}}^{>}(\omega) \\
& =\int \frac{d \omega}{2 \pi}\left(2-e^{-i \omega t}-e^{i \omega t}\right) \frac{2}{1-e^{-\beta \omega}} \operatorname{Im} G_{\tilde{O} \tilde{O}}^{R}(\omega) \\
& \simeq \int \frac{d \omega}{2 \pi}\left(2-e^{-i \omega t}-e^{i \omega t}\right) \frac{2 q^{2} \Gamma T}{\omega^{2}} \sim q^{2} \Gamma T t .
\end{aligned}
$$

In the last step, we approximate the integrand using (5.1). This is justified as the dominant contribution at large $t$ comes from small $\omega$. This gives the long time behavior of the fluctuation. The linear dependence in $t$ shows it is a random walk growth, which is unstable. Note that the scaling relation holds in the regime $\omega \ll T$, which sets the time scale of the unstable mode. In other words, on a time scale $t \sim \frac{1}{T}$, a fluctuation effectively destroys the D-instanton background. Therefore, we conclude that the lifetime of the instanton is set by the temperature. The conclusion is in line with the classic field theory results that the large instanton is suppressed at high temperature $[20,21]$. As a final remark, we stress that classic results were obtained based on thermodynamics consideration; our conclusion were obtained from an analysis of real time fluctuations. 


\section{ACKNOWLEDGMENTS}

S. L. is grateful to Yan Liu and Nan Su for valuable discussions. S. L. is in part supported by the One Thousand Talent Program for Young Scholars and NSFC under Grant No. 11675274 and the NSFC Key Project under Grant No. 11735007. S. W. L. is in part supported by Fudan University and the One Thousand Talent Program for Young Scholars.

\section{APPENDIX: HOLOGRAPHIC RENORMALIZATION}

The purpose of this appendix is to express correlation functions in terms of elements of the response matrix. The derivation closely follows $[24,28]$. We start from the 5D Euclidean action (3.3) with Gibbon-Hawking term $S_{G H}$,

$$
\begin{aligned}
S_{E}= & S_{5 \mathrm{D}}+S_{G H}, \\
= & \frac{1}{\kappa_{5}^{2}} \int d^{5} x \sqrt{g_{(5)}}\left[\mathcal{R}^{(5)}-\frac{1}{2}(\partial \Phi)^{2}+\frac{1}{2} e^{2 \Phi}(\partial \chi)^{2}+12\right] \\
& +\frac{2}{\kappa^{2}} \int d^{4} x \sqrt{h} K .
\end{aligned}
$$

Following [29], we add an additional counterterm $S_{C T}$ to cancel the volume divergence to the bulk theory which is

$$
S_{C T}=-\frac{3}{\kappa_{5}^{2}} \int d^{4} x \sqrt{h}
$$

Note that this is the counterterm for the gravity sector. Other counterterms for dilaton and axion are also needed; see [30] and references therein for early constructions. We will need additional counterterms in the discussion below. We assume the boundary is taken at $r=\Sigma$ which would be sent to infinity at the end of the calculation. Using the equations of motion in (3.5) and keeping the contribution from the boundary $r=\Sigma$, and combining the resultant boundary terms with $S_{G H}$ and $S_{C T}$, we can obtain

$$
\begin{aligned}
S_{E}= & \frac{1}{2 \kappa_{5}^{2}} \int d^{4} x\left[\frac{3 r^{4} h_{00}^{2}}{4 f^{3 / 2}}\left(1-f^{1 / 2}\right)+\frac{9 r^{4} H h_{00}}{2 f^{1 / 2}}\left(f^{1 / 2}-1\right)\right. \\
& -\frac{9 r^{4} H^{2}}{4}\left(f^{1 / 2}-1\right)-\frac{3 r^{5} f^{\prime} H h_{00}}{8 f}+\frac{3 r^{5} f^{\prime} H^{2}}{8} \\
& -\frac{1}{4} r^{5}\left(h_{00}+3 f H\right) \delta \Phi \Phi^{\prime}+\frac{1}{4} e^{\Phi} r^{5}\left(h_{00}+3 f H\right) \delta \chi \Phi^{\prime} \\
& +e^{\Phi} r^{5} f \delta \Phi \delta \chi \Phi^{\prime}+\frac{3}{4} r^{5}\left(H h_{00}\right)^{\prime}+\frac{3}{2} r^{5} f H^{\prime} H \\
& \left.-\frac{1}{2} r^{5} f \delta \Phi(\delta \Phi)^{\prime}+\frac{1}{2} e^{2 \Phi} r^{5} \delta \chi(\delta \chi)^{\prime}\right] .
\end{aligned}
$$

Plugging the asymptotic expansions (3.11) into the above formula, we obtain the on-shell action,

$$
\begin{aligned}
S_{E}= & \frac{1}{2 \kappa_{5}^{2}} \int d^{4} x\left[\left(-\frac{3 a_{1} b_{0}}{2}-c_{0} c_{1}+f_{0} f_{1}\right) r^{2}+\frac{3}{8} a_{0}^{2}\right. \\
& -\frac{15}{4} a_{0} b_{0}-3 a_{2} b_{0}+\frac{3}{8} b_{0}^{2}-3 a_{0} b_{2}-6 b_{0} b_{2}-c_{1}^{2} \\
& -2 c_{0} c_{1}+\frac{1}{2} c_{0} c_{h}+f_{1}^{2}+2 f_{0} f_{2}-\frac{1}{2} f_{0} f_{h} \\
& +q\left(-a_{0} c_{0}-3 b_{0} c_{0}+a_{0} f_{0}+3 b_{0} f_{0}-4 c_{0} f_{0}\right) \\
& \left.-16\left(c_{0} c_{h}-f_{0} f_{h}\right) \ln r+\cdots\right] .
\end{aligned}
$$

Note that all the coefficients are functions of $t: a_{0}=a_{0}(t)$ etc. To evaluate the correlators in momentum space, we express $S_{E}$ in terms of Fourier components of the coefficient $a_{0}(k)$. In doing this, we find that the coefficients of the superficially $r^{2}$ divergent terms are of the type $\omega_{E}^{2} b_{0}^{2}$, $\omega_{E}^{2} c_{0}^{2}$, and $\omega_{E}^{2} f_{0}^{2}$. Similarly, the $\ln r$ divergent terms are of the type $\omega_{E}^{4} c_{0}^{2}$ and $\omega_{E}^{4} f_{0}^{2}$. Therefore all divergent terms are contact terms and thus should be discarded [31]. The remaining action is given by

$$
\begin{aligned}
S_{E}= & \frac{1}{2 \kappa_{5}^{2}(2 \pi)} \int d \omega_{E}\left[-3 b_{0}\left(-\omega_{E}\right) a_{2}\left(\omega_{E}\right)-6 b_{0}\left(-\omega_{E}\right) b_{2}\left(\omega_{E}\right)-2 c_{0}\left(-\omega_{E}\right) c_{2}\left(\omega_{E}\right)+2 f_{0}\left(-\omega_{E}\right) f_{2}\left(\omega_{E}\right)\right. \\
& +\frac{3}{8}\left[a_{0}\left(-\omega_{E}\right) a_{0}\left(\omega_{E}\right)-10 a_{0}\left(-\omega_{E}\right) b_{0}\left(\omega_{E}\right)+3 b_{0}\left(-\omega_{E}\right) b_{0}\left(\omega_{E}\right)\right] \\
& \left.+q\left[-a_{0}\left(-\omega_{E}\right) c_{0}\left(\omega_{E}\right)-3 b_{0}\left(-\omega_{E}\right) c_{0}\left(\omega_{E}\right)+a_{0}\left(-\omega_{E}\right) f_{0}\left(\omega_{E}\right)+3 b_{0}\left(-\omega_{E}\right) f_{0}\left(\omega_{E}\right)-4 c_{0}\left(-\omega_{E}\right) f_{0}\left(\omega_{E}\right)\right]\right] .
\end{aligned}
$$

There are three types of terms in (A5). The first type is the product of sources and VEVs like $b_{0}\left(-\omega_{E}\right) a_{2}\left(\omega_{E}\right)$. The second type is the product of sources like $a_{0}\left(-\omega_{E}\right) a_{0}\left(\omega_{E}\right)$,

\footnotetext{
${ }^{3}$ To simplify the calculation, we have set the 5D cosmological $\Lambda=-6$ and assume all the fields depend on $\tau$ and $r$ only, which is case of our interest.
}

which indicates the presence of constant terms in the resulting correlators. The third type is the product of sources and $q$, such as $q a_{0}\left(-\omega_{E}\right) c_{0}\left(\omega_{E}\right)$. Now we use the response matrix to express VEVs in terms of sources. We can then proceed to calculate the correlators by the differentiation of the action with respect to sources. 


$$
\begin{aligned}
& G_{00,00}^{E}=\int d \tau d^{3} x e^{i \omega_{E} \tau}\left\langle T_{E}^{00}(\tau, x) T_{E}^{00}(0)\right\rangle \\
& =4 \frac{(2 \pi) \delta^{2} S_{E}}{\delta a_{0}\left(-\omega_{E}\right) \delta a_{0}\left(\omega_{E}\right)}, \\
& G_{00, i i}^{E}=\int d \tau d^{3} x e^{i \omega_{E} \tau}\left\langle T_{E}^{00}(\tau, x) T_{E}^{i i}(0)\right\rangle \\
& =4 \frac{(2 \pi) \delta^{2} S_{E}}{\delta a_{0}\left(-\omega_{E}\right) \delta b_{0}\left(\omega_{E}\right)}, \\
& G_{i i, j j}^{E}=\int d \tau d^{3} x e^{i \omega_{E} \tau}\left\langle T_{E}^{i i}(\tau, x) T_{E}^{j j}(0)\right\rangle \\
& =4 \frac{(2 \pi) \delta^{2} S_{E}}{\delta b_{0}\left(-\omega_{E}\right) \delta b_{0}\left(\omega_{E}\right)}, \\
& G_{00, O}^{E}=\int d \tau d^{3} x e^{i \omega_{E} \tau}\left\langle T_{E}^{00}(\tau, x) O_{E}(0)\right\rangle \\
& =2 \frac{(2 \pi) \delta^{2} S_{E}}{\delta a_{0}\left(-\omega_{E}\right) \delta f_{0}\left(\omega_{E}\right)}, \\
& G_{00, \tilde{O}}^{E}=\int d \tau d^{3} x e^{i \omega_{E} \tau}\left\langle T_{E}^{00}(\tau, x) \tilde{O}_{E}(0)\right\rangle \\
& =2 \frac{(2 \pi) \delta^{2} S_{E}}{\delta a_{0}\left(-\omega_{E}\right) \delta c_{0}\left(\omega_{E}\right)}, \\
& G_{i i, O}^{E}=\int d \tau d^{3} x e^{i \omega_{E} \tau}\left\langle T_{E}^{i i}(\tau, x) O_{E}(0)\right\rangle \\
& =2 \frac{(2 \pi) \delta^{2} S_{E}}{\delta b_{0}\left(-\omega_{E}\right) \delta f_{0}\left(\omega_{E}\right)},
\end{aligned}
$$

$$
\begin{aligned}
G_{i i, \tilde{O}}^{E} & =\int d \tau d^{3} x e^{i \omega_{E} \tau}\left\langle T_{E}^{i i}(\tau, x) \tilde{O}_{E}(0)\right\rangle \\
& =2 \frac{(2 \pi) \delta^{2} S_{E}}{\delta b_{0}\left(-\omega_{E}\right) \delta c_{0}\left(\omega_{E}\right)}, \\
G_{O O}^{E} & =\int d \tau d^{3} x e^{i \omega_{E} \tau}\left\langle O_{E}(\tau, x) O_{E}(0)\right\rangle \\
& =\frac{(2 \pi) \delta^{2} S_{E}}{\delta f_{0}\left(-\omega_{E}\right) \delta f_{0}\left(\omega_{E}\right)}, \\
G_{\tilde{O} \tilde{O}}^{E} & =\int d \tau d^{3} x e^{i \omega_{E} \tau}\left\langle\tilde{O}_{E}(\tau, x) \tilde{O}_{E}(0)\right\rangle \\
& =\frac{(2 \pi) \delta^{2} S}{\delta c_{0}\left(-\omega_{E}\right) \delta c_{0}\left(\omega_{E}\right)}, \\
G_{O \tilde{O}}^{E} & =\int d \tau d^{3} x e^{i \omega_{E} \tau}\left\langle O_{E}(\tau, x) \tilde{O}_{E}(0)\right\rangle \\
& =\frac{(2 \pi) \delta^{2} S}{\delta f_{0}\left(-\omega_{E}\right) \delta c_{0}\left(\omega_{E}\right)} .
\end{aligned}
$$

We have suppressed the overall prefactor $\frac{1}{2 \kappa_{5}^{2}}$ for notational simplicity. Note that the prefactor has mass dimension four. We restore dimension by multiplying $(\pi T)^{4}$ and converting the prefactor to the field theory quantity $\frac{(\pi T)^{4}}{2 \kappa_{5}^{2}}=\frac{\pi^{2} N^{2} T^{4}}{8}=P$, which is the same as pressure of plasma. Recall that we have confirmed the response matrix is even in $\omega_{E}$. This nice property allows us to treat the sources as ordinary numbers. This is essentially the procedure adopted in [24]. When the response matrix is not even in $\omega_{E}$, a more careful treatment is needed [28]. Plugging (3.18) into (A6), we obtain (4.1).
[1] T. Schäfer and E. V. Shuryak, Instantons in QCD, Rev. Mod. Phys. 70, 323 (1998).

[2] D. J. Gross, R. D. Pisarski, and L. G. Yaffe, QCD and instantons at finite temperature, Rev. Mod. Phys. 53, 43 (1981).

[3] D. Diakonov, N. Gromov, V. Petrov, and S. Slizovskiy, Quantum weights of dyons and of instantons with nontrivial holonomy, Phys. Rev. D 70, 036003 (2004).

[4] D. Diakonov and V. Petrov, Confining ensemble of dyons, Phys. Rev. D 76, 056001 (2007).

[5] Y. Liu, E. Shuryak, and I. Zahed, Confining dyon-antidyon Coulomb liquid model. I., Phys. Rev. D 92, 085006 (2015).

[6] Y. Liu, E. Shuryak, and I. Zahed, Light quarks in the screened dyon-antidyon Coulomb liquid model. II., Phys. Rev. D 92, 085007 (2015).

[7] Y. Liu, E. Shuryak, and I. Zahed, Instanton-dyon liquid model. III. Finite chemical potential, Phys. Rev. D 94, 105011 (2016).
[8] Y. Liu, E. Shuryak, and I. Zahed, Light adjoint quarks in the instanton-dyon liquid model IV., Phys. Rev. D 94, 105012 (2016).

[9] Y. Liu, E. Shuryak, and I. Zahed, Instanton-dyon liquid model. V. Twisted light quarks, Phys. Rev. D 94, 105013 (2016).

[10] E. Poppitz, T. Schäfer, and M. Unsal, Continuity, deconfinement, and (super) Yang-Mills theory, J. High Energy Phys. 10 (2012) 115.

[11] E. Poppitz, T. Schäfer, and M. Ünsal, Universal mechanism of (semi-classical) deconfinement and theta-dependence for all simple groups, J. High Energy Phys. 03 (2013) 087.

[12] H. Liu and A. A. Tseytlin, D3-brane D instanton configuration and $N=4$ super-YM theory in constant self-dual background, Nucl. Phys. B553, 231 (1999).

[13] J. L. F. Barbon and A. Pasquinucci, Aspects of instanton dynamics in AdS/CFT duality, Phys. Lett. B 458, 288 (1999). 
[14] K. Ghoroku, T. Sakaguchi, N. Uekusa, and M. Yahiro, Flavor quark at high temperature from a holographic model, Phys. Rev. D 71, 106002 (2005).

[15] B. Gwak, M. Kim, B.-H. Lee, Y. Seo, and S.-J. Sin, Holographic D instanton liquid and chiral transition, Phys. Rev. D 86, 026010 (2012).

[16] Z.-q. Zhang, D.-f. Hou, and G. Chen, Heavy quark potential and jet quenching parameter in a D-instanton background, Eur. Phys. J. A 52, 357 (2016).

[17] Z.-q. Zhang, D.-f. Hou, and G. Chen, Imaginary potential of moving quarkonia in a D-instanton background, J. Phys. G 44, 115001 (2017).

[18] D. Mateos and D. Trancanelli, Thermodynamics and instabilities of a strongly coupled anisotropic plasma, J. High Energy Phys. 07 (2011) 054.

[19] L. Cheng, X.-H. Ge, and S.-J. Sin, Anisotropic plasma at finite $U(1)$ chemical potential, J. High Energy Phys. 07 (2014) 083.

[20] R. D. Pisarski and L. G. Yaffe, The density of instantons at finite temperature, Phys. Lett. 97B, 110 (1980).

[21] E. V. Shuryak and M. Velkovsky, The instanton density at finite temperatures, Phys. Rev. D 50, 3323 (1994).

[22] G. W. Gibbons, M. B. Green, and M. J. Perry, Instantons and seven-branes in type IIB superstring theory, Phys. Lett. B 370, 37 (1996).
[23] A. Kehagias and K. Sfetsos, On asymptotic freedom and confinement from type IIB supergravity, Phys. Lett. B 456, 22 (1999).

[24] G. Policastro, D. T. Son, and A. O. Starinets, From AdS/CFT correspondence to hydrodynamics. 2. Sound waves, J. High Energy Phys. 12 (2002) 054.

[25] G. D. Moore and M. Tassler, The sphaleron rate in SU(N) gauge theory, J. High Energy Phys. 02 (2011) 105.

[26] D. T. Son and A. O. Starinets, Minkowski space correlators in AdS/CFT correspondence: Recipe and applications, J. High Energy Phys. 09 (2002) 042.

[27] M. Le Bellac, Thermal Field Theory (Cambridge University Press, Cambridge, 2011).

[28] M. Kaminski, K. Landsteiner, J. Mas, J. P. Shock, and J. Tarrio, Holographic operator mixing and quasinormal modes on the brane, J. High Energy Phys. 02 (2010) 021.

[29] H. Liu and A. A. Tseytlin, $D=4$ super-Yang-Mills, $D=5$ gauged supergravity, and $D=4$ conformal supergravity, Nucl. Phys. B533, 88 (1998).

[30] M. Gutperle and W. Sabra, Instantons and wormholes in Minkowski and (A)dS spaces, Nucl. Phys. B647, 344 (2002).

[31] P. Kovtun and A. Starinets, Thermal Spectral Functions of Strongly Coupled $N=4$ Supersymmetric Yang-Mills theory, Phys. Rev. Lett. 96, 131601 (2006). 